\title{
Design on Knitwear Fashion
}

\author{
Jing Gao \\ School of Art and Garment \\ Tianjin Polytechnic University \\ Tianjin 300384, China
}

\begin{abstract}
Knitwear fashion owns both the characters of knitted garments and fashion. Its unique fabrics, colors, shapes and technology, as well as casualness and comfortableness to wear, attract people's hearts on beauty and it becomes popular rookie. This paper, based on the designer's point of view, through market research, focuses on the analysis of the characteristics of knitwear fashion, fabric choices, color matching and fashion trend, which highlights the significance of knitwear fashion in today's society as well as the quick development of knitted fabrics whose roles could not be ignored.
\end{abstract}

Keywords: Knitwear fashion, Color, Fabric, Fashion trend

China's garment enterprises, after accumulating considerable energy, have gradually entered into the world. Garment is the mirror that reflects the images of a country and a nation. Around the world, no matter in what kind of culture or economy background, we can find garments that match it. In current era, the beautifying function of garments is completely shown. The garments could make an ordinary girl into a beauty in an instant moment. No wonder the love of women on garments is so lasting and I am afraid that the charm of clothing lies in this.

Knitwear is a new member of garment world. Now, it is playing a more and more important role in our lives and becomes an indispensable part of our lives. It, based on knitted underwear, makes full use of the extension character of knitted fabric and the plasticity of raw materials, inherits and develops the traditional processing technology for traditional knitwear. Its good elasticity, good sense of touching, and casualness becomes popular in fashion. Someone predicts that knitwear fashion will become popular. At present, knitwear fashion develops quickly and has found a lace in the world garment forests.

Knitted garments include knitted sweaters, knitted sportswear, knitwear fashion, knitted underwear, knitted T-shirt and knitted accessories. Knitwear fashion refers to all kinds of knitted fashion cloth made out of knitted fabric. The styles of knitwear fashion are diverse. There are knitwear suits that are for good to use and are of fashion. It is natural and comfortable. Also, there are knitwear fashions which pay attention to personal style and taste and highlight the unique design.

Commonly used knitwear fashion fabric includes cotton, chemical fiber, and silk and polyester cover cotton.

After doing research on knitted fabric fashion in the market, it is found that fall and winter knitwear feature in their natural appearance, comfortable touch sense. They emphasize natural fibers, elastic fiber, functional use of a new type of fiber and gives excellent comfort and functionality to knitwear; or it adopts lightweight, mid-hollow fibers so that it could maintain the warmth-keeping function and reduce the weight of knitwear so that it tends to be light; yarn is more delicate and more exquisite; its organizational structure is richer, stresses the complex and integrated use of various structure, and forms a rich fabric appearance. The functional settling technology with the organizational structure enables it to have moisture absorbing, air flowing, warmth keeping, healthy keeping, safety, easy-to-care functions.

The followings are some popular fabrics.

Acetel has the unique properties the same as silk. Its fiber luster, bright colors, drape and touching sense are al excellent. The knitting fabric with it feels smooth and comfortable to wear, is breathable moisture, and is of light texture and low moisture regain, is not easy to play ball, and is anti-static. The woven knit georgette, cornflakes, and other fabrics made of acetel won consumer's preference. 
Modal fiber is a new type of environmentally friendly fiber, which has the features the comfortableness of cotton, viscose of the drape, the strength of polyester and silk sense. What is more, after being washed for many times, it could still maintain their softness and bright color. Knitting technology still combines the fabric and softness and high-elastic characteristics of fabric so that the two can complement each other's superiority. In the circular knitting machine, it adopts Modal and spandex together. It is soft, smooth, flexible, floating in the air hanging, of beautiful sheen, of moisture permeability and silky with the hand. Fashion made of this kind of fabrics could at maximum, reflect the curve of the human body, shows the sculpture of a female carcass and sexy charm, is the high taste apparel favored by people.

Environmentally friendly renewable green knit fabric, Lyocell, Tencel and spandex are knitted together to form knitted flat needle organization (Han $\mathrm{Bu}$ ), rib, double-rib (cotton). Their texture is soft. Their surface is flat and velvet. They have good flexibility. Their products have elegant style and have the appearance of silk. Their drape, breathability and water stability are good. Hence, they are the ideal high-grade fabric for tight epidemic fashion, casual wear and sportswear.

Flash knitted fabrics have the effect of flash and have always been a favorite of fashion designers. In the circular knitting machine, it adopts golden and silver raw materials and other raw materials, which are intertwined. The surface of the materials have strong reflecting flashing effect or adopt gold-plated approach, in knitted fabric on the Flash emergence of a variety of patterns effect to form the flashing effect on the knitting surface. While the back of the materials are flat, soft and comfortable Hence, it is good knitting garment materials. If you use this kind of knitting materials to design female fashion and evening dress, it could, through the eye-catching fabrics flash and romantic style, show brilliant knitted fabric, beautiful charm, all-round performance to knitting clothing style in order to provide product development broad prospects.

Feature knitted fabric has four trench Coolmax fibers and it can quickly transfer human beings' sweat to the surface of the clothes and then rapidly evaporate it so that it could keep skin cool and comfortable. It has good moist transmitting function. The knitted fabric with cotton fiber has excellent moist transmitting effect and is widely used in making T-shirts, sportswear and so on.

In order to show the characteristics of various fabric and to expand the space for garment design, knitwear fashion often uses fabric weave pattern changes, color changes and other methods to enrich the practical functions and appearances of garments, such as the clothes in the cuffs, collar and lap Department flexibility to be able to set good rib cloth; In the place where air is needed, it applies mesh cloth. Or it uses printed flowers to replace knitted jacquard commonly used in fashion, and so on. It uses the organizational structure of jacquard, mesh, and changes to form rich and changing jacquard thin strip surface. After taking out yarn, the entire knitting garment looks light and graceful. It also advanced computer jacquard technique and organizational structure change to form tousy lines on surface, which is special old style.

Color is one of the elements of the beauty. In the three elements that constitute garment, fabric, color and style, color is the most eye-catching, the most active and most sensitive. It can be said that color is the soul of the garment. To study fashion knitwear, it is essential to discuss the meaning of color. Through the exploration of color, we can increase our understanding of color, master the use of the color in the most expressive way and it is of incalculable value for product design and development.

As the commonest aesthetic form and content, color is used in each aspect of human beings' life. In knitwear fashion, no matter it is the design of style or the matching of accessories, color is necessary.

The selection of knitwear fashion colors includes the designers' selection on color and the wearers' selection on color. The designers should consider the overall situation and choose different colors so as to match different wearers. The wears could choose proper colors according to their tastes.

The matching of knitwear fashion colors could adopt same color matching or upper light and lower deep or lower light and upper deep, so that the viewers could feel good. Due to age, sex, and the aesthetic taste difference, people's favorite colors are different. This requires us, in our design, to pay attention to the matching of apparel fabrics, color, shape and size, skin color, and characters. We should use simple methods, with minimal color, to design garments that more comply with the aesthetic tastes of the times.

Fashion, under current background, is no longer people's focus point but has become the necessity of live and is gradually accepted by people because fashion reflects the potential consumption and market.

Fashion represents an overall image sense and the basic elements and features that constitute these images. The fundamental origin and base of fashion forecast is life body. It represents people's potential needs and change trend of moods through popular colors, fabrics, and styles. Both the fabric and clothing has a lot of emotional factors, such as the vertical sense, feeling soft sense, smooth sense, concave and protruding sense, transparent sense, light perception, and a sense of folding, and so on. We have elegant, cold and beautiful, pastoral, science fictional, or pure and ladies of leisure, or solemn clothes. Emotional factors are added into yarn, fabrics, and clothing, is a distillation of the material 
value, is a reflection of art and is the base of the added values. The annual trends are actually from the emotional material with the theme of the beginning. The color theme is of the more emotional characteristics. It gives people a sentiment and its effect is the most direct appeal and is the most powerful visual impact. It embodies through color yarn and fabrics. The mechanism of the appearance of fabric is very important emotional factor, especially in the 1990s, texture, the mechanism of the development of a sense of old and new fabric has become an important symbol. The $1990 \mathrm{~s}$ is a complex mixture with the times, a variety of fibers mixed in the past to break the simple combination of fiber. The sci-fi flash effect is more practical demonstrated for the proliferation, and to play down the penetration in the fabric of all species. A large number of man-made fibers occupy larger proportions. In addition, the woven fabric structure change is like a million flowers posing unpredictable results. Each change gives people a subtle move. The texture of fabrics of different combinations creates clothing but also a wealth of space. Garments, as comprehensive end-products, integrate the emotional factors including colors, yarns, fabrics, accessories, and accessories.

As a result, popularity is no longer a popularity of style or a type. It also includes colors, fabrics, styles and many other elements, which together forms the overall style. It is not only the pilot for new product design and development, but also is the embodiment of modern live culture and consumption fashion.

In recent years, seeing from the popular clothing trend, the change of context of life and society trend exerts influence on yarn, fabric and styles. Knitwear fashion introduces the styles that emphasize on ecological balance and at the same time, it brings out the natural, peaceful and gentle feeling of the wearers. For the color fashion, like the world's nostalgic mood, it also advocates sun faded and after- washing color and effect. Based on this trend, a variety of chemical fiber emerges one after another; a variety of sand-washed fabrics and styles as well as its mood of nostalgia won consumers' love.

Due to the changes of styles, many people do not wear those clothes that are still good because they want to follow the fashion. At this stage, we should not be enthralled by the colorful and changing garments. The key point is to have your own style.

\section{References}

Lei. (2001). Knitwear Fashion Design. Beijing: China Knitting Press.

Li, Wenjie. (1990). Knitwear Fashion Design. Light Industry Press.

Liu, Xiaogang \& Huang, Yuanqiang. (1991). Fashion Design Introduction. Shanghai: Shanghai Culture Press.

Wu, Tianxiang \& Wang, Gang. (1991). Novel Knitting Fabric Fashion Cutting and Making. Shanghai: Shanghai Science and Technology Press. 